\title{
THE MAGNETIC SPECIFIC HEAT OF THE BEG MODEL
}

\author{
M. Gzik-Szumiata and T. Balcerzak \\ Department of Solid State Physics, University of Łódź \\ Pomorska 149/153, 90-236 Łódź, Poland
}

\begin{abstract}
The magnetic contribution to the specific heat of the Blume-EmeryGriffiths model with spin-one is studied by the cluster variational method in pair approximation. The nearest neighbour correlation functions are taken into account. The temperature dependencies of the specific heat are calculated and discussed in the context of the phase diagrams obtained recently for the thin film with bilayer geometry.
\end{abstract}

PACS numbers: 75.10.-b, 75.70.-i

\section{Introduction}

The Blume-Emery-Griffiths (BEG) model for spin $S=1$ has been extensively studied on account of very rich phase diagrams it exhibits [1-4]. Besides ordinary exchange interactions the model takes into account the single-ion anisotropy and biquadratic terms. These interactions allow us to discuss many interesting properties, for instance the existence of multicritical points, continuous and discontinuous phase transitions, staggered quadrupolar and ferrimagnetic phases, as well as the re-entrant phenomenon.

Cluster variational method in pair approximation (CVMPA) is one of the most accurate and convenient method for studies of the BEG model $[2,4,5]$. As far as the bilayer system is concerned, CVMPA has not been applied yet for the calculations of the magnetic specific heat, although it has been used for studies of the phase diagrams [4] and magnetic susceptibility [5].

For the above reasons, the aim of the present paper is investigation of the specific heat within CVMPA for the bilayer system with $z=5$. Starting from the Gibbs energy, the theoretical formula for the specific heat has been derived. On this basis, we have performed the numerical calculations of the specific heat curves vs. temperature, for various kinds of the phase diagrams. The numerical results, selected for some characteristic cases, are illustrated in figures and discussed. 


\section{Theory}

The BEG Hamiltonian is of the form

$$
\mathcal{H}=-J \sum_{\langle i j\rangle} S_{i} S_{j}-A \sum_{\langle i j\rangle} S_{i}^{2} S_{j}^{2}-D \sum_{i} S_{i}^{2}-h \sum_{i} S_{i},
$$

where $S_{i}=0, \pm 1 ; J$ and $A$ denote the bilinear and biquadratic exchange constant, whereas $D$ is the single-ion anisotropy constant and $h$ stands for an external field. We will consider the nearest neighbour (nn) interactions only and a system consisting of two interpenetrating sublattices $a$ and $b[3-5]$.

For the identification of various magnetic phases, we must find the sublattice magnetisations, $m_{\alpha} \equiv\left\langle S_{i, \alpha}\right\rangle$, and the quadrupolar moments, $q_{\alpha} \equiv\left\langle S_{i, \alpha}^{2}\right\rangle$, where $\alpha=a, b$. In particular, for $J>0$ and $h=0$, the following four phases are possible:

ferromagnetic phase (F): $m_{a}=m_{b} \neq 0, q_{a}=q_{b}$;

paramagnetic phase (P): $m_{a}=m_{b}=0, q_{a}=q_{b}$;

staggered quadrupolar phase (SQ): $m_{a}=m_{b}=0, q_{a} \neq q_{b}$;

ferrimagnetic phase (I): $0 \neq m_{a} \neq m_{b} \neq 0, q_{a} \neq q_{b}$.

The occurrence of these phases depends on the values of $A$ and $D$ parameters, as well as the temperature, hence various phase diagrams can be obtained.

As far as CVMPA is concerned, we will refer to the paper [4] where the details of the method have been given, and the full expression for the Gibbs energy has been presented. Having Gibbs energy, $G$, the magnetic contribution to the specific heat, $C_{h}$, can be obtained from the general formulae

$$
C_{h}=-T\left(\frac{\partial^{2} G}{\partial T^{2}}\right)_{h}=T\left(\frac{\partial S}{\partial T}\right)_{h}=\left(\frac{\partial H}{\partial T}\right)_{h},
$$

where $S$ is the entropy of the system, and $H$ denotes the enthalpy. The latter is defined as the mean value of the Hamiltonian (1), i.e., $H \equiv\langle\mathcal{H}\rangle$.

The final formula for the specific heat, however, is too long to be presented in this paper and will be published elsewhere. It is a complicated function of $m_{\alpha}$ and $q_{\alpha}$, as well as the nearest neighbour pair correlation functions $\left\langle S_{i} S_{j}\right\rangle,\left\langle S_{i}^{2} S_{j}\right\rangle,\left\langle S_{i} S_{j}^{2}\right\rangle$, and $\left\langle S_{i}^{2} S_{j}^{2}\right\rangle$. All these functions can be found when corresponding variational parameters are known, which, in turn, have to be determined from the cluster variational equations [4]. In this paper we will present only some of numerical results, i.e., the specific heat curves vs. temperature, which are characteristic of the various magnetic phases $F, P, S Q$, and I.

\section{The numerical results and discussion}

The numerical calculations were performed for the bilayer system with $z=5$ and for $h=0$. The specific heat has been studied for various kinds of the phase diagrams obtained in [4].

For instance, in Fig. 1a the phase diagram $k_{\mathrm{B}} T / J$ vs. $D / J$ for $A / J=-0.5$ is presented [4]. The continuous and discontinuous phase transitions, represented by solid and dashed line, respectively, meet at the tricritical point (the bold dot). The regions of ferromagnetic and paramagnetic phases are indicated.

In relation to Fig. 1a, in Fig. 1b the specific heat was calculated for $A / J=$ -0.5 and $D / J=-1.24$. In this case three phase transitions occur, i.e. $\mathrm{F} / \mathrm{P}$ (discontinuous, at $k_{\mathrm{B}} T / J=0.342$ ), $\mathrm{P} / \mathrm{F}$ (continuous, re-entrant, at $k_{\mathrm{B}} T / J=0.634$ ) 


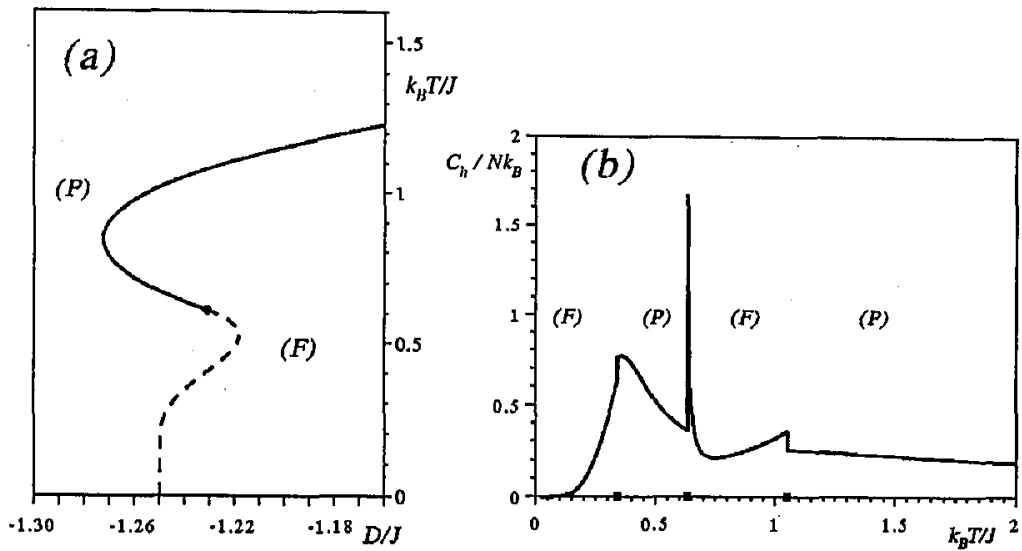

Fig. 1. (a) The phase diagram for $A / J=-0.5$ (see Ref. [4]). (b) The magnetic specific heat per one lattice site for $A / J=-0.5$ and $D / J=-1.24$.

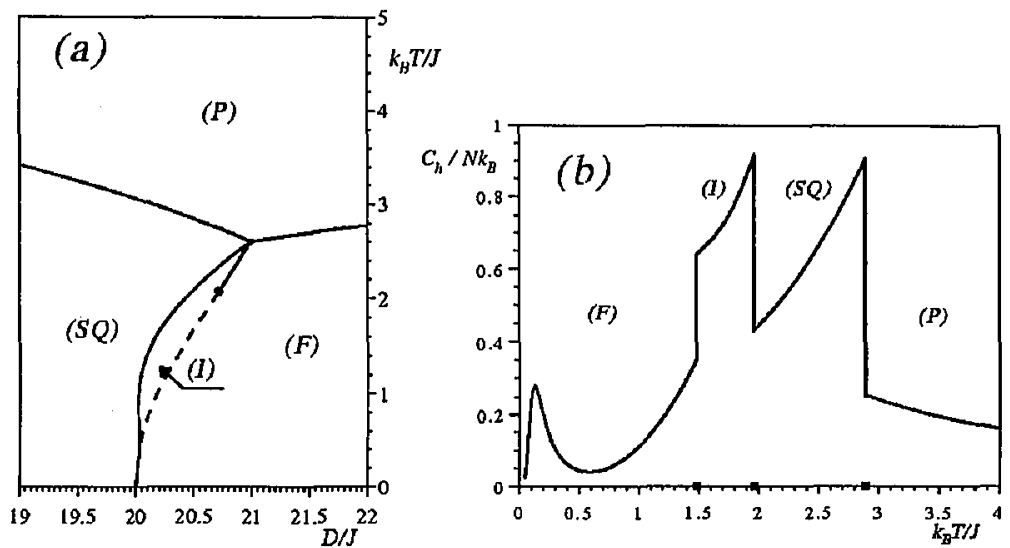

Fig. 2. (a) The phase diagram for $A / J=-5$ (see Ref. [4]). (b) The magnetic specific heat per one lattice site for $A / J=-5$ and $D / J=20.4$.

and $\mathrm{F} / \mathrm{P}$ (ordinary, continuous, at $k_{\mathrm{B}} T / J=1.051$ ). The temperatures of these phase transitions are marked by the dots on the horizontal axis. We can see the rapid changes of the specific heat at the phase transition temperatures, especially the high peak at the re-entrant temperature, $k_{\mathrm{B}} T / J=0.634$, is remarkable.

In Fig. 2a we present the phase diagram $k_{\mathrm{B}} T / J$ vs. $D / J$ for $A / J=-5[4]$. In this case four phases (P, F, SQ, and I) are seen. All these phases meet at the point of their coexistence (i.e. tetracritical point), at which the four continuous phase transition lines meet at different angles. The discontinuous (dashed line) phase transitions between $\mathrm{F}$ and I phase meet the continuous ones at the tricritical point (the bold dot).

In relation to Fig. $2 \mathrm{a}$, to perform the specific heat calculations we chose the value $D / J=20.4$ (Fig. $2 \mathrm{~b}$ ). The phase transition temperatures: $\mathrm{F} / \mathrm{I}$ (discon- 
tinuous, at $k_{\mathrm{B}} T / J=1.478$ ), I/SQ (continuous, at $k_{\mathrm{B}} T / J=1.965$ ) and $\mathrm{SQ} / \mathrm{P}$ (continuous, at $k_{\mathrm{B}} T / J=2.890$ ) are marked by the dots on the horizontal axis (Fig. 2b). The jumps of the specific heat at the phase transition temperatures are seen. The small maximum appearing in the low temperature region is not attributed to the phase transition. Its existence can be explained by analysis of the magnetisation curve in ferromagnetic phase.

All the results presented here are the stable solutions corresponding to the minimum of the Gibbs energy. Let us note that for low temperatures the specific heat always tends to zero, which is in agreement with the third principle of thermodynamics. Thus, CVMPA proved to be a good technique for studies of not only the phase diagrams [4], or the magnetic susceptibility [5], but of the specific heat as well.

Although CVMPA is known to be a much better technique than the molecular field approximation (for instance, for the Bethe lattices it gives the exact results [4]), as far as the regular lattices are concerned, some verification of the results by other, more accurate methods, would be desirable. For instance, it would be of a great value to perform the Monte Carlo simulations for the bilayer system in the frame of the same model.

\section{References}

[1] M. Blume, V.J. Emery, R.B. Griffiths, Phys. Rev. A 4, 1071 (1971).

[2] J.W. Tucker, J. Magn. Magn. Mater. 71, 27 (1987).

[3] A.Z. Akheyan, N.S. Ananikian, J. Phys. A, Math. Gen. 29, 721 (1996).

[4] J.W. Tucker, T. Balcerzak, M. Gzik, A. Sukiennicki, J. Magn. Magn. Mater. 187, 381 (1998).

[5] M. Gzik-Szumiata, T. Balcerzak, Acta Phys. Slov. 48, 611 (1998). 